\title{
Eosinophilic esophagitis" (EoE) do we need diagnostic guideline update??
}

\begin{abstract}
EoE guidelines have been first published in 2007 and updated in 2011. The main motive for the update was the description of a new entity closely related to EoE under the name of "PPI- responsive Eosinophilic Esophagitis" (PPI-REE) after the publication of first guidelines. The two guidelines, however, remained almost the same in their main diagnostic steps apart from the emphasis on differentiation of PPI-REE as a distinct sub entity from EoE in the new guidelines. They both ignored two important issues; standardization of the size of hpf of the microscope to avoid errors in cell counting and emphasis on the value of biopsies from other parts of gut at least in research studies. We came to the conclusion, after a short review of the various guidelines that some update is needed and some questions have to be answered more clearly in both in clinical and research fields.
\end{abstract}

Volume 2 Issue 5 - 2015

\section{Hussein Abdel Hamid}

Department of Gastroenterology and Hepatology, President of the African Middle East Association of Gastroenterology, Egypt

Correspondence: Hussein Abdel-Hamid, Department of Gastroenterology and Hepatology, President of the African Middle East Association of Gastroenterology, Egypt Email hussein.egypt22@gmail.com

Received: : June 15, 2015 | Published: September 8, 2015
Abbreviations: REE, responsive eosinophilic esophagitis; EC, eosinophilic colitis; EGE, eosinophilic gastroenteritis; GERD, gastroesophageal reflux disease

\section{Introduction}

EoE has recently become under focus. It was traditionally thought to be a rare disease with prevalence less than $1 \%$ of the general population in many studies. ${ }^{1,2}$ However, it is by no means rare in the endoscopy units where its prevalence jumps to 5-16\% of patients undergoing upper endoscopy. ${ }^{3-5}$ This simply means that this entity should always be put in mind in patients with esophageal symptoms as dysphagia (up to $100 \%$ ), acute food impaction (50\% of emergency cases), heartburn (10-100\%) and less commonly NCCP. All these symptoms are non-specific and occur in such diseases as achalusia, GERD and peristaltic abnormalities. It is believed that the incidence of EoE has increased twenty three fold in the last 30 years but it is quite unknown weather this marked increase is real or simply due to increased awareness or due to both. EoE is the commonest of a family known as Eosinophilic GI disorders which include, in addition, Eosinophilic gastroenteritis (EGE) and Eosinophilic colitis (EC). As Eosinophilic GI disorders may involve any pant of gut from the esophagus to the rectum, EoE may occur alone or in association with other Eosinophilic lesions in the gut. EoE, however, most commonly occurs as an isolated lesion, at least according to our present state of knowledge.

\section{Definition}

$\mathrm{In}^{1}$ Liacouras defined EoE as a chronic inflammatory condition characterized by esophageal dysfunction, diffuse Eosinophilic infiltration of the esophageal mucosa and absence of other causes of esinophilia. It is thus considered a kind of chronic Eosinophilic inflammation related to asthma and other allergic conditions. It is thought to be an immune-mediated disease triggered by food or an environmental antigen stimulating. The immuno-inflammatory response. ${ }^{6}$

\section{First diagnostic guidelines}

EoE was first described as an entity in 1993 but it was only in 2007 when its first guidelines were published as follows:

1) Symptoms related to esophageal dysfunction.
2) Diffuse Eosinophilic infiltration of the esophageal mucosa (at least 15 esinophils / hpf in at least one esophageal biopsy).

3) Exclusion of GERD either by a negative PPI test or by negative $\mathrm{pH}$ metry. Other causes of esinophilia apart from GERD should be also excluded.

4) It was stressed that the symptoms and the endoscopic appearances of EoE are all non-specific, not to mention that normal endoscopy is found in $7-10 \%$ of cases. ${ }^{7,8}$ Esophageal esinophilia occurs in other conditions as GERD, Crohn's disease, achalusia , malignancy, connective tissue disorders, food allergy and infectious ${ }^{6,7}$ For this reason diagnosis of EoE depends on Eosinophilic cell count and not on the mere presence of esinophils in the mucosa. It is clear that the guidelines focused on exclusion of GERD in particular probably because it was the commonest of the list of the differential diagnosis.

\section{Discovery of PPI-responsive esophageal esinophilia (PPI-REE)}

After publication of the first guidelines, PPI-REE was described as a new entity or sub entity which was identical to EoE in its clinical, endoscopic and histological features. It differs only in its good response to PPI although it was not associated with acid reflux. The mechanism of this therapeutic response remains unknown. It is not precisely known weather PPI-REE is a variant of EoE, a variant of GERD or a totally new entity. It was clear after this new advance that modified guidelines are needed

\section{Updated guidelines 20 I I (6)}

I. Symptoms related to esophageal dysfunction.

II. Diffuse Eosinophilic infiltration of the esophageal mucosa (at least 15 esinophils/ hpf in at least one esophageal biopsy) with few exceptions.

III. Esinophilia isolated to the esophagus.

IV. Exclusion of other causes of esophageal esinophilia particularly PPI-REE by PPI therapeutic test which became a mandatory diagnostic step. (40my BID for 2 weeks). The focus on exclusion of PPI-REE was because it can easily be misdiagnosed as EoE and because it was found to constitute up to $30 \%$ of EoE family. ${ }^{9,10}$ 


\section{Evaluation of the guidelines}

Guidelines 2007 and guidelines 2011 agree almost completely on the definition of EoE and the main diagnostic steps. They differed only in the main differential diagnosis that has to be carefully excluded. While guidelines 2007 stressed on GERD exclusion either by PPI test or pH-metry, guidelines 2011 stressed on exclusion of the new entity PPI-REE by PPI test. The option of pH-metry was not considered in this respect. On the other hand, both guidelines ignored two important issues, the first is the need for a standard lens power to avoid counting errors, and the second is the need to take biopsies from other parts of gut to avoid missing co-existent Eosinophilic lesions. Since the costeffectiveness of routine biopsies from other parts of gut is unknown at the present time, we recommend this procedure to be routine only in research studies to begin with. Later on this practice may become popularized if found cost effective. This differentiation between isolated EoE and EoE associated with Eosinophilic gastroenteritis is important because it has a bearing on the therapeutic strategies. In addition, we believe that pH-metry should not to be excluded completely from the updated guidelines as it is still important in differentiating of PPI-REE from GERD. Other issues of controversy include the diagnostic value of systemic allergic diseases and the potential role of capsule endoscopy.

\section{Standardization of the lens}

Various studies support the concept of standardization of the size of hpf of lens which depends on the model and the maker of the microscopes. It varies from 0.12 to $0.44 \mathrm{~mm}$. It is possible that a count that falls below the diagnostic threshold in one microscope may be above the threshold in a different one. ${ }^{11}$ The lack of consensus on the size of hpf, can result in 23 fold variability in the description of esinophil density and therefore can affect the diagnosis and the follow-up. ${ }^{12}$

\section{Gastro duodenal biopsies}

To my mind, researchers working on EoE should take biopsies at least from the stomach and the duodenum in addition to the esophagus and so should be aware of the diagnostic guidelines of Eosinophilic gastroenteritis (EGE) which comes next to EoE in prevalence. The guidelines of EGE states that the diagnosis depends on infiltration of the mucosa by dense aggregates or sheets of esinophilis, with potential extension into the muscularis mucosa and submucosa, and with esinophil count $>30 / \mathrm{hpf}$ in at least in $5 \mathrm{hpfs} .{ }^{13,14}$ It is clear that the Eosinophilic count in EGE is much higher than in EoE because the gastric and the bowel mucosae normally contain esinophils but the normal esophageal mucosa does not.

\section{Eosinophilic colitis}

This is the least common of the family of Eosinophilic GI disorders. Since no guidelines have been published so far $^{15}$ we do not recommend routine biopsies of the colon in clinical practice. Diagnosis depends on the presence of excess Eosinophilic in the colon more than in other parts of gut.

\section{Conclusion}

YES. We need guidelines update especially for research studies.
The new guidelines should not ignore standardization of the lens and gastro duodenal biopsies. pH-metry should not be ignored as it has a place in differention of PPI-REE from GERD.

\section{Acknowledgements}

None.

\section{Conflicts of interest}

The authors declare that there is no conflict of interest.

\section{References}

1. Furuta GT, Liacouras CA, Collins MH, et al. Eosinophilic esophagitis in children and adults: a systematic review and consensus recommendations for diagnosis and treatment. Gastroenterology. 2007;133(4):1342-1363.

2. Gonsalves N, Ritz S, Yang GY, et al. A prospective clinical trial of allergy testing and food elimination diet in adults with eosinophilic esophagitis. Gastroenterology. 2007;132:A6.

3. Ronkainen J, Talley NJ, Aro P, et al. Prevalence of esophageal eosinophils and eosinophilic esophagitis in adults: the population-based Kalixanda study. Gut. 2007;56(5):615-620.

4. Prasad GA, Talley NJ, Romero Y, et al. Prevalence and predictive factors of eosinophilic esophagitis in patients presenting with dysphagia: a prospective study. Am J Gastroenterol. 2007;102(12):2627-2632.

5. Veerappan GR, Perry JL, Duncan TJ, et al. Prevalence of eosinophilic esophagitis in an adult population undergoing upper endoscopy: a prospective study. Clin Gastroenterol Hepatol. 2009;7(4):420-426.

6. Liacouras CA, Furuta GT, Hirano I, et al. Eosinophilic esophagitis: updated consensus recommendations for children and adults. $J$ Allergy Clin Immunol. 2011;128(1):3e6-20e6.

7. Dellon ES. Diagnosis and management of eosinophilic esophagitis. Clin Gastroenterol Hepatol. 2012;10(10):1066-1078.

8. Matzinger MA, Daneman A. Esophageal involvement in eosinophilic gastroenteritis. Pediatr Radiol. 1981;13(1):35-38.

9. Dranove JE, Horn DS, Davis MA, et al. Predictors of response to proton pump inhibitor therapy among children with significant esophageal eosinophilia. J Pediatr. 2009;154(1):96-100.

10. Dohil R, Newbury RO, Aceves S. Transient PPI responsive esophageal eosinophilia may be a clinical sub- phenotype of pediatric eosinophilic esophagitis. Dig Dis Sci. 2012;57(5):1413-1419.

11. Dellon ES, Aderoju A, Woosley JT, et al. Variability in diagnostic criteria for eosinophilic esophagitis: a systematic review. Am J Gastroenterol. 2007;102(10):2300-2313.

12. Parfitt JR, Gregor JC, Suskin NG, et al. Eosinophilic esophagitis in adults: distinguishing features from gastroesophageal reflux disease: a study of 41 patients. Mod Pathol. 2006;19(1):90-96.

13. Klein NC, Hargrove RL, Sleisenger MH, et al. Eosinophilic gastroenteritis. Medicine (Baltimore). 1970;49(4):299-319.

14. Talley NJ, Shorter RG, Phillips SF, et al. Eosinophilic gastroenteritis: a clinicopathological study of patients with disease of the mucosa, muscle layer, and subserosal tissues. Gut. 1990;31(1):54-58.

15. Abdul Rahman A. Eosinophilic colitis: epidemiology, clinical features, and current management. Ther Adv Gastroenterol. 2011;4(5):301-309. 